\title{
ACOUSTICS2008/731 \\ On the separation of hydrodynamic and acoustic waves in linear free-shear flows
}

\author{
A. Agarwal and G. Gabard \\ Institute of Sound and Vibration Research, University of Southampton, Highfield, SO17 1BJ Southampton,
} UK

The governing equations for sound propagation through free-shear flows, like jets and mixing layers, are the linearized Euler equations. These equations support both hydrodynamic and acoustic waves. For an aeroacoustician wishing to study the refraction effects of a sound source by shear flows, it is important to distinguish the acoustic solution from the hydrodynamic waves. Agarwal et al. (AIAA J., vol. 42, no. 1, 2004) presented a technique to achieve this in the frequency domain. In this talk, we present a time-domain technique to separate the hydrodynamic and acoustic waves. The idea is to implement a filter that filters out only the acoustic wave solution from the linearized Euler equations. Some sample solutions are presented for two-dimensional free-shear flows and comparisons are made against known analytical solution for parallel flows and from solution obtained by approximate methods, which have a limited range of applicability. The advantage for the present technique is that it is applicable to arbitrary frequencies and flow profiles.

Keywords:

Technical area: Noise (NS) - Euronoise

PACS \#1: 43.28.Ra Generation of sound by fluid flow, aerodynamic sound and turbulence

PACS \#2:

PACS \#3: 